\title{
Was macht einen Coach zum Coach? Weiterbildung und Qualifizierung
}

\author{
Eric Lippmann
}

Online publiziert: 7. Oktober 2015

(C) Die Autor(en) 2015. Dieser Artikel ist auf Springerlink.com mit Open Access verfügbar.

\begin{abstract}
Zusammenfassung In diesem Artikel wird der Frage nachgegangen, was denn eine gute Qualifizierung zum Coach ausmacht. Zuerst werden dazu Anforderungen an Coach und Coaching-Weiterbilder/innen beschrieben und mit den wichtigsten Wirkfaktoren im Coaching in Zusammenhang gestellt. Darauf aufbauend geht es um Anforderungen an die Coaching-Weiterbildungen, um am Schluss der Frage nachzugehen, was denn professionelle Coachs für ihre Weiterqualifizierung brauchen.
\end{abstract}

Schlüsselwörter Coaching-Weiterbildung ·

Qualifizierung · Professionalisierung

\section{What makes a difference? Further education and qualification for a professional coach}

\begin{abstract}
This article gives some answers to the question, what a good qualification programme for a professional coach should contain: Which are the demands for a good basic qualification programme and what do professional coaches need for their own further qualification?
\end{abstract}

Keywords Further education for coaching - Qualification programme for coaching · Professional Coaching

E. Lippmann $(\bowtie)$

Institut für Angewandte Psychologie, ZHAW,

Pfingstweidstrasse 96 ,

Postfach 707, 8037 Zürich, Schweiz

E-Mail: eric.lippmann@zhaw.ch

\section{Einleitung}

Wo bleibt der Coaching-Bericht der Stiftung Warentest? fragten Böning und Fritschle 2008 in „Coaching fürs Business“ und dachten dabei primär an die Kunden, welche Coaching als Dienstleistung einkaufen. Acht Jahre später liegt ein Bericht vor. Allerdings nicht bezogen auf die Anbieter von Coaching, sondern von Coaching-Weiterbildungen. Es wird da gefragt, was denn eine gute Einstiegsqualifizierung bieten sollte, um für einen Markt gewappnet zu sein, der in den letzten Jahren im deutschen Sprachraum boomte. Der Markt ist nicht nur gewachsen, er ist dabei auch noch unübersichtlicher geworden. Im deutschen Sprachraum schätzt man, dass von ca. 11.300 Business-Coachs etwa 7100 Führungskräfte-Coachs aktiv sind. Gemäß verschiedener Studien haben fast vier Fünftel von ihnen ein akademisches Studium und etwa gleich viele eine Coaching-Ausbildung absolviert, und immerhin auch 30\% eine therapeutische Zusatzausbildung (Winkler et al. 2013). Grund beruhigt zu sein? Das hängt davon ab, ob man das Glas als halb voll oder halb leer betrachtet, einer Metapher, auf die am Schluss dieses Artikels nochmals kurz eingegangen werden soll. Zumindest als nicht unbedenklich kann die Tatsache angesehen werden, dass rund ein Viertel der Coachs gar keine bzw. gemäß Vogelauer (2013b) eine nur bis 20 Tage dauernde Coaching-Weiterbildung aufführt. Wie kann man feststellen, ob ein Coach ein Coach ist? Ist eine absolvierte Weiterbildung eher ein Garant als ein akademisches Studium? Und ist eine längere Weiterbildung besser als eine kürzere? Wer eine/n qualifizierte/n Coach sucht, steht somit vor ähnlichen Auswahlproblemen wie Personen, welche sich zum Coach qualifizieren möchten. Die Auswahl ist Groß und die Weiterbildungsangebote sind sehr heterogen. Böning und Fritsche (2008) beschreiben die Suche nach einem Weiterbildungs-Anbieter für ihre Mitarbeitenden und kommen zum Schluss: „Wer als Kunde 
diesbezüglich wenig Vorkenntnisse und Hartnäckigkeit mitbringt, läuft Gefahr, entweder irgendwann im Suchprozess aufzugeben oder - möglicherweise noch schädlicher für das Image der Szene - an den falschen zu geraten" (S. 164). Man könnte auch etwas zynisch bemerken, dass wer eine qualifizierte Coaching-Weiterbildung sucht, sich dafür ein Coaching gönnen sollte, um die Chance zu verringern, an die falschen zu geraten. Doch für die Suche nach dem entsprechenden Coach wäre vielleicht auch wieder ein Coaching nötig. Der Zynismus begründet sich nicht zuletzt aus der Tatsache, dass einige Coachs und Coach-Qualifizierer zu einem guten Teil auch von der Coaching-Weiterbildung leben, so dass Kühl von einem „Kettenbriefsystem“ spricht (2006, S. 11). Dies mag mit ein Grund sein, dass es so zahlreiche Angebote gibt. Die Datenbank von Christopher Rauen enthält gut 200 Weiterbildungsgänge. Der DBVC schätzt, dass pro Jahr allein in Deutschland etwa 4000 Personen eine Coaching-Weiterbildung absolvieren. Wie viele dann tatsächlich als Coach in der Folge tätig sind, bleibt offen. Auf jeden Fall kann mit Sicherheit gesagt werden, dass nicht alleine die Qualifizierungen entscheidend sind, ob jemand zum erfolgreichen Coach wird. Aber bereits während einer Weiterbildung zeigt sich erfahrungsgemäß, wer höhere Chancen auf einen späteren Erfolg als Coach haben wird. Dies insbesondere dann, wenn während der Qualifizierung mehr als nur ein Mandat nachgewiesen werden muss, wie es in diesem Artikel empfohlen wird. Hier soll der Frage nachgegangen werden, was denn eine gute Weiterbildung ausmacht. Zuerst werden dazu Anforderungen an Coach und Coach-Weiterbilder/innen beschrieben und mit den wichtigsten Wirkfaktoren im Coaching in Zusammenhang gestellt. Darauf aufbauend geht es um Anforderungen an die Coaching-Weiterbildungen, um am Schluss der Frage nachzugehen, was denn - im doppelten Sinn - danach kommt: Wie qualifizieren sich professionelle Coachs weiter und wie geht es weiter mit Coaching-Weiterbildungen in der Zukunft?

\section{Anforderungen an Coach und Coach-Weiterbilder/in und entsprechende Wirkfaktoren}

Im Zentrum der Weiterbildung steht die Frage, welche Kompetenzen zu erwerben sind, um als Coach über genügend Ressourcen zu verfügen. Der Kompetenzbegriff wird seit Mitte der 1990er Jahre in verschiedenen Wissenschaftsdisziplinen diskutiert. Allerdings hat sich bis anhin noch keine allgemein akzeptierte Definition von Kompetenz herausgebildet (vgl. z. B. Kauffeld 2006). In Anlehnung an Konzepte der Selbstorganisationstheorie kann Kompetenz umschrieben werden als die Fähigkeit und Bereitschaft, in offenen, komplexen und dynamischen Situationen selbstorganisiert, zielgerichtet, aufgabengemäss, situationsbedingt verantwortungsbewusst zu handeln. Berufliche Handlungskompetenz wird häufig als Gegenbegriff zu Qualifikation aufgefasst. Die Aneignung einer Qualifikation ist in der Regel an ein zuvor definiertes Ausbildungsziel und entsprechendes Curriculum gebunden und wird mit einem Zeugnis bestätigt. Eine Qualifikation kann eine Grundlage für die Entwicklung beruflicher Handlungskompetenz darstellen, aber ist noch kein Garant für selbstorganisiertes und kreatives Handeln. Erst wenn durch die Handlung deutlich wird, was ein Mensch wirklich kann und weiss, wird von Kompetenz gesprochen (Erpenbeck und von Rosenstiel 2007). Berufliche Handlungskompetenz wie es Coaching darstellt, wird pragmatisch in die vier Kompetenzklassen Fach-, Methoden-, Sozial- und Persönlichkeits-/ Selbstkompetenz untergliedert (vgl. z. B. Kauffeld 2006; Meier und Janssen 2011; Merz und Frey 2011). Dabei ist zu berücksichtigen, dass die Klassen interpendent und daher nicht immer klar voneinander abgrenzbar sind.

Aus dem Konzept der Handlungskompetenz folgt die Vorstellung, dass die Weiterbildung zum Coach auf der Basis eines „ganzheitlichen Lernens“ erfolgt. Das heißt, dass die Entwicklung aller vier Kompetenzen insgesamt und gleichberechtigt gefördert werden muss. Für die Beratungstätigkeit ist es schliesslich das praktische Tun und Handeln mit den entsprechenden Erfahrungen, die einen nachhaltigen Lernprozess auslösen können.

Im Folgenden werden anhand der vier Felder einige zentrale Kompetenzen in Stichworten aufgeführt, die eine Coaching-Weiterbildung weiterentwickeln soll (vgl. Abb. 1: Die 4 Dimensionen der Handlungskompetenz):

\begin{tabular}{lll}
\hline Fachkompetenzen & & \\
\hline Grundlagenwissen & Beratungswissen & $\begin{array}{l}\text { Wissen aus fachver- } \\
\text { wandten Gebieten }\end{array}$ \\
\hline $\begin{array}{l}\text { Grundlagen der } \\
\text { Psychologie }\end{array}$ & $\begin{array}{l}\text { Supervision und } \\
\text { Coaching (Theorien }\end{array}$ & $\begin{array}{l}\text { Ethik } \\
\text { (Arbeits-) }\end{array}$ \\
$\begin{array}{l}\text { Arbeits- und Organi- } \\
\text { sationspsychologie, } \\
\text { Klinische Psycholo- } \\
\text { gie und psychothera- } \\
\text { peutische Richtungen }\end{array}$ & $\begin{array}{l}\text { Organisationsbera- } \\
\text { tung und -ent- }\end{array}$ & $\begin{array}{l}\text { Soziologie } \\
\text { Erkennen von } \\
\text { wirtschaftlichen, }\end{array}$ \\
$\begin{array}{l}\text { Betriebswirtschaftli- } \\
\text { ches Wissen }\end{array}$ & Teamberment & politischen und \\
Management, & -entwicklung und & Entwicklung-en \\
Leadership und & Problembearbeitung & \\
Rollenkonzepte zur & und -lösung & \\
Führung & Konfliktberatung & \\
& Projektberatung & \\
& Diagnose in & \\
& unterschiedlichen & \\
\hline
\end{tabular}

\begin{tabular}{ll}
\hline Methodenkompetenzen & \\
\hline Phasen der Beratung & Interventionen und Methoden \\
\hline Auftrags- und Rollenklärung & Zielbezogenheit bei den \\
Kontrakt & Interventionen \\
Diagnose & Einsatz von Methoden und \\
Prozessgestaltung & Tools \\
Architektur und Design & Moderations- und \\
& Präsentationstechniken \\
& Evaluation \\
\hline
\end{tabular}


Abb. 1 Die 4 Dimensionen der Handlungskompetenz

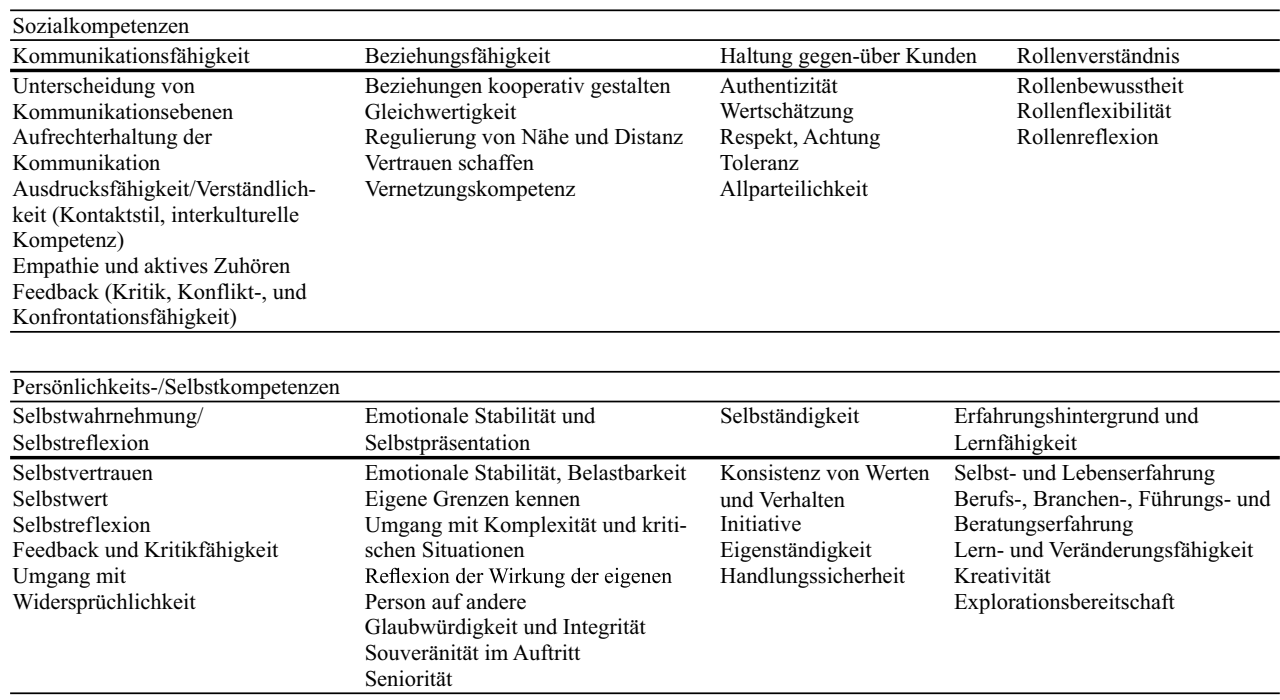

Die hier genannten Kompetenzen sind nicht vollständig und variieren je nach Weiterbildungsangebot. Idealerweise definiert ein Anbieter einer Coaching-Weiterbildung zu Beginn eines Lehrganges, welchen Ausprägungsgrad bezüglich der einzelnen Kompetenzen jemand mitbringen und welches Niveau beim Abschluss erreicht werden sollte. Dies ist natürlich abhängig von der Art der Zertifizierung bzw. Diplomierung.

Welche Aspekte den ausgebildeten Coachs bzw. den Coaching-Kunden wichtig sind, sei beispielhaft aus drei Untersuchungen ergänzend aufgeführt. Aus der Sicht der Coachs sind folgende Kompetenzen unter den „Top Ten“ genannt (Kuchen und Pedrun 2006, S. 70):

1. Selbstreflexion

2. Selbst- und Lebenserfahrung

3. Aktiv und unvoreingenommen zuhören können

4. Authentizität (Echtheit, Glaubwürdigkeit)

5. Transparente Informationsgestaltung bei Auftrags- und Kontextklärung

6. Psychologisches Wissen

7. Fähigkeit, Beziehungen kooperativ zu gestalten

8. Beratungserfahrung

9. Vertrauensvolle Atmosphäre schaffen

10. Wissen der Organisationspsychologie

Bei befragten Organisationen werden aus Kundensicht folgende „Top Ten“ genannt (Brandenberger und Gassmann 2006, S. 40):

1. Authentizität (Echtheit)

2. Aktiv und unvoreingenommen zuhören

3. Vernetztes Denken

4. Zielorientierung

5. Wertefreiheit/Neutralität

6. Methodenvielfalt und diese flexibel einsetzen können

7. Rollendefinition bei der Auftragsklärung
8. Klare Zielsetzungen vereinbaren

9. Empathie

10. Feedback und Kritikfähigkeit

Bei der gemischten Befragung von Vogelauer (2013b, S. 166) sind folgende acht Anforderungen in dieser Reihenfolge am bedeutendsten:

1. Verschwiegenheit, Loyalität

2. Genanntes aufgreifend, situativ einstellend

3. Auf Ziel und Ergebnis orientiert

4. Empathisch und auf Beziehung orientiert

5. Breite Lebenserfahrung

6. Strukturiert, geordnet, klares Konzept

7. Lässt Kunden Raum, hält sich zurück

8. Feld- und Fachkenntnis

Während die ersten beiden Befragungen in der Schweiz durchgeführt wurden ( $N=167$ Coachs bzw. 84 Organisationen), sind unter den rund 100 Antwortenden bei Vogelauer etwa ein Drittel Kunden, knapp 20\% Personalentwickler und die Hälfte Coachs (von allen gut die Hälfte aus Österreich und je ca. ein Fünftel aus Deutschland und der Schweiz). Es kann gut sein, dass die Differenzen der Gewichtungen unter anderem durch die unterschiedlichen Rollen und Kulturen entstehen.

\subsection{Vergleich mit den Wirkfaktoren im Coaching}

Vergleicht man die aufgeführten Kompetenzen mit den Wirkfaktoren, welche die Forschung für Coaching als relevant bezeichnen, so fallen hier auch große Überschneidungen auf. In Anlehnung an die von Grawe erforschten Schulen übergreifenden allgemeinen Wirkprinzipien für die Psychotherapie ist Greif der Frage nachgegangen, in wie weit diese auf Coaching übertragbar sind (Greif 2009). Methodisches Ziel war, Wirkfaktoren herauszuarbeiten, die 
anhand von Video- oder Tonbandaufzeichnungen von Coaching-Sitzungen durch trainierte Beobachter eingeschätzt werden können. Greif geht davon aus, dass nicht die angewandten Methoden für ein erfolgreiches Coaching entscheidend sind, sondern die Orientierung an den Wirkfaktoren. Er unterscheidet sieben allgemeine Wirkfaktoren, an denen sich eine Coaching Weiterbildung orientieren kann. Im Curriculum empfiehlt es sich, vor allem Methoden zu vermitteln, welche geeignet sind, die verschiedenen Faktoren zu unterstützen:

1. Wertschätzung und emotionale Unterstützung des Klienten durch den Coach

Dieser Faktor enthält die von Carl Rogers bereits als zentral erachteten Basisvariablen Empathie (emotionale Unterstützung) und Wertschätzung, die der Coach dem Kunden gegenüber zum Ausdruck bringt. Diese Aspekte sind auch klar in den erwähnten Umfragen häufig aufgeführt.

\section{Affektaktivierung und -kalibrierung}

In Anlehnung an die neuropsychologische Motivationsund Persönlichkeitstheorie von Kuhl (2001) geht Greif davon aus, dass rationales Denken und der Zugang zum Selbst durch starke Affekte erschwert werden. Deshalb soll der Kunde seinen Affekten Ausdruck verleihen (z. B. „Luft ablassen“ bei Ärger als Affektkalibrierung). Dazu kann der Coach den Kunden beispielsweise zum bewussten nachdenken anregen durch eine Frage wie: „Welche Gefühle haben Sie in dieser Situation?“. Dadurch wird der Kunde eingeladen, sich in den entsprechenden Modus zu begeben, so dass sich der Affekt in der Regel abschwächt.

3. Ergebnisorientierte Problemreflexion

Darunter versteht Greif die Analyse und Reflexion über die Wahrnehmung der Problemsituation des Kunden, die der Coach so anregt, dass aus den Reflexionen konkrete Folgerungen für die Zukunft abgeleitet werden können. Dazu werden häufig Frage- oder Rollenspieltechniken angewandt, welche Perspektivenwechsel des Kunden bezogen auf die Problemsituation fördern. Greif betont in Anlehnung an Gassmann und Grawe (2006), dass die Problemlösungen wirksamer aktiviert werden, wenn Problemaktualisierungen der Aktivierung der positiven Ressourcen vorausgehen. Eine ähnliche Sichtweise vertritt auch Schmidt in seinem hypnosystemischen Ansatz, indem er in Abgrenzung zum rein lösungsorientierten Ansatz nach de Shazer für eine „Liebesaffäre zwischen Problem und Lösung“ plädiert (ausführlicher dazu Schmidt 2013a).

4. Ergebnisorientierte Selbstreflexion
Ergebnisorientierte Selbstreflexion werden vom Coach als Prozesse angeregt, bei denen der Kunde über seine Werte, Eigenschaften, Stärken, Schwächen, sein individuelles Verhalten und Erleben bewusst nachdenkt. Diese Reflexion führt optimalerweise zu neuen Einsichten oder Pläne zur Veränderung von Empfindungen, Einstellungen und Handlungen. Damit unterscheidet sich ergebnisorientierte Reflexion von „ziellosem Grübeln“ einerseits, und von unreflektiertem, blindem Aktionismus andererseits. Die Methoden und Fähigkeiten, solche Selbstreflexionen zu fördern, bezeichnen Greif et al. (2012) als „besondere professionelle Qualifizierung von Psychotherapeuten und Coaches" (Greif 2008, S. 383).

\section{Zielklärung}

Die Klärung und Herausarbeitung von durch den Kunden bearbeitbaren Zielen ist ein zentraler Aspekt im Coaching. Die Reflexion der persönlichen Motive kann unter die Förderung der Selbstreflexion zugeordnet werden. Sie bildet eine wichtige Basis für die konkrete Beschreibung und Definition der Ziele des Kunden. Dabei ist es wichtig, bei den dazugehörenden Erwartungen immer auch den Kontext miteinzubeziehen und zu schauen, welche Auswirkungen die Erreichung von Zielen im relevanten Umfeld des Kunden zu erwarten sind. Ziele müssen aber nicht immer scharf nach den sogenannten SMART-Kriterien formuliert werden. Gerade bei Einstieg kann es sinnvoll sein, mit vagen Zielvorstellungen zu starten. Erfahrungsgemäß verändern sich Ziele im Verlauf eines Coachingprozesses, deshalb ist es zentral, regelmäßig eine Zwischenstandortbestimmung zu machen, um Zielkongruenzen herzustellen und Veränderungen bzw. bisher erarbeitete Schritte sichtbar zu machen.

\section{Ressourcenaktivierung}

Damit wird umschrieben, dass durch ein Coaching dem Kunden seine internen Ressourcen (Kompetenzen, Wissen, Erfahrungen, Eigenschaften, Potenziale usw.) wie auch seine externen (z. B. andere Personen, Institutionen und andere „Quellen“ usw.) bewusster werden. Durch die Grundhaltung des Coach, dass der Kunde „kundig“ und damit „Experte seiner selbst“ bleibt, ist der Aspekt der „Hilfe zu“" seitens des Coachs sehr zu relativieren. Angesprochen sind hier vielmehr Interventionen aus der ressourcen- und lösungsorientierten Beratung wie auch aus der Positiven Psychologie, welche die Potenziale des Kunden optimal unterstützen können.

\section{Umsetzungsunterstützung}

Bezüglich dieses Wirkfaktors stützt sich Greif auf Grawe, der die „Problembewältigung“ in drei Schritten beschreibt (Gassmann und Grawe 2006): Lösungsentwicklung und handlungsorientierte Umsetzung (Trans- 
fer) in der Praxis und Unterstützung des Kunden bei der Zielerreichung. Da es sich beim Coaching häufig um kurzfristige, ergebnisorientierte Unterstützung handelt, führt Greif entsprechende Methoden an, welche dazu geeignet sind:

„Shadowing“, bei dem der Coach den Kunden in der Umsetzungssituation begleitet und mit ihm anschlieBend sein Verhalten und die Auswirkungen im Kontext reflektiert (Kaufel et al. 2006). Virtuelles Transfer-Coaching, welches Geissler (2011) beschreibt kann ebenso unterstützend und im Aufwand geringer sein als das Telefon-Shadowing (Greif 2008).

\section{Anforderungen an eine Coaching Weiterbildung}

Wie einleitend erwähnt, hat die Stiftung Warentest 2013 Gütekriterien entwickelt für Einstiegsqualifizierungen zum Coach. Die Gütekriterien der Stiftung Warentest wurden über eine schriftliche Befragung bei Anbietern von Coaching-Weiterbildungen erhoben. Dazu kamen Ableitungen aus den empfohlenen Standards gemäß Webseiten verschiedener Coaching-Verbände. Der so erstellte Kriterienkatalog wurde anschließend mit Coaching-Experten aus Wissenschaft und Praxis und mit Vertretern von Coaching-Verbänden diskutiert. Der Deutsche Bundesverband Coaching (DBVC 2012) äußert sich in seinem Kompendium ebenfalls ausführlich zur Aus- und Weiterbildung. Die Anforderungen des DBVC sind etwas tiefer als bei den Einstiegsqualifizierungen zum Coach, sie liegen deutlich unter den Kriterien des Berufsverbandes für Coaching, Supervision und Organisationsberatung (BSO 2013) in der Schweiz oder der Deutschen Gesellschaft für Supervision (DGSv 2013). Im Folgenden sind hauptsächlich aus diesen Quellen die Anforderungen abgeleitet, die an eine Coaching Weiterbildung gestellt werden sollten.

\subsection{Formale Voraussetzungen}

Der DBVC definiert einen Mindestumfang von 150 Zeitstunden für ein Curriculum von 12-18 Monaten, der Österreichische Dachverband für Coaching 190. Die Stiftung Warentest empfiehlt $250 \mathrm{~h}$ Präsenzunterricht, während der $\mathrm{BSO}$ von 450 dozentenbegleiteten Stunden (inkl. mindestens $40 \mathrm{~h}$ Lehrsupervision) ausgeht. Ein Lehrgang dauert gemäß BSO minimal 2 Jahre, mindestens 20 Tage sollten in einer konstanten Gruppe absolviert werden. Die DGSv (2013) verlangt sogar $500 \mathrm{~h}$ Präsenzunterricht und $90 \mathrm{~h}$ eigene praktische Tätigkeit.

Die ideale Gruppengrösse wird mit 10-15 Personen angegeben, umfasst die Gruppe mehr als 15 Personen, empfiehlt die Stiftung Warentest die Präsenz mindestens zweier
Dozierenden. Von Vorteil ist es, wenn wenigstens einer der Dozierenden den Lehrgang begleitet.

Es ist empfehlenswert, wenn ein Weiterbildungsinstitut die Qualifizierungen regelmäßig durchführt, Rauen und Steinhübel (2005) fordern gar eine jährliche Kadenz, die allerdings bei längerdauernden Qualifizierungen anspruchsvoll werden dürfte. Auf jeden Fall evaluiert ein Anbieter seine Leistungen regelmäßig nach einem schlüssigen Konzept, das dann in die Weiterentwicklung des Curriculums einfließt.

Die Eingangsqualifizierung sollte klar definiert werden. Idealerweise verfügen Interessenten über ein Hochschulstudium, es sollten aber auch Personen mit Berufserfahrung und anderen Qualifikationen aufgenommen werden können. Der BSO verlangt beispielsweise bereits $30 \mathrm{~h}$ Erfahrungen als Kunde. Eine heterogene Zusammensetzung einer Gruppe kann insofern von Vorteil sein, als in der Organisationswelt auch sehr unterschiedliche Personen als Kunden Coaching in Anspruch nehmen. Die Bereitschaft, sich mit Diversität auseinanderzusetzen, ist genau so wichtig wie Fähigkeit zur Selbstreflexion, zur Auseinandersetzung mit sich und andern Teilnehmenden. Deshalb muss das Auswahlverfahren auch zum Ergebnis kommen können, dass sich ein Interessent nicht für die Qualifizierung eignet. Entsprechend sollten solche Kandidaten auch beraten werden, ob und wie sie nach Einschätzung des Anbieters die noch fehlenden Eingangsvoraussetzungen erwerben können.

\subsection{Ziel}

Das Ziel einer Coaching-Qualifizierung soll sein, dass ein Teilnehmer am Ende ein ihm gemäßes Coaching-Konzept entwickeln kann. Eine entsprechende Rahmentheorie bildet die Basis, damit Absolventen in der Lage sind, Coachings im beruflichen Kontext qualifiziert durchzuführen. Wie weit ein Curriculum Individualisierungen bei den Lernzielen zulässt, ist für jeden Lehrgang speziell zu prüfen. Der DBVC empfiehlt dazu, dass Anbieter und Weiterbildungsteilnehmer hierüber einen transparenten Dialog führen (2012). Aus eigener Erfahrung denke ich jedoch, dass einer zu hohen Individualisierung Grenzen gesetzt sind. Gewisse Eingangskompetenzen muss jemand mitbringen, allenfalls können neben der Weiterbildung unterstützende Maßnahmen hilfreich sein, dass jemand trotz fehlender Vorkenntnisse die Weiterbildungsziele auch erreichen kann (z. B. zusätzlicher Wissenserwerb in einzelnen Fachgebieten, Zusatzkurs im Verfassen von Arbeiten, Selbsterfahrungsoder Therapiegruppen usw.).

\subsection{Inhalte}

Die Inhalte lassen sich aus den erforderlichen Kompetenzen eines Coachs ableiten. Sie sind selbstverständlich abhängig 
von der Dauer, der Zielgruppe und vom Anbieter. Wenn sich eine Coaching-Weiterbildung beispielswiese exklusiv an Psychologinnen und Psychologen richtet, so wird das Curriculum ganz anders aussehen, als wenn es für Personen aus betriebswirtschaftlichem oder naturwissenschaftlichem Hintergrund gedacht ist.

Es empfiehlt sich, dass eine Weiterbildung auf unterschiedlichen, wissenschaftlich anerkannten Denkrichtungen und „Schulen“ aufgebaut wird mit entsprechenden methodenübergreifenden Beratungsansätzen. Dies begünstigt bei den Absolventen einen breiteren Zugang zum Fachgebiet, Perspektivenwechsel sind dadurch einfacher möglich und die Gefahr von doktrinärem, einseitigem Denken kann minimiert werden. Eine spezifische Vertiefung in einer Richtung (z. B. lösungsorientiert, systemisch, Gestalt) kann dann immer noch später erfolgen, denn ein Coach sollte sich schliesslich permanent weiterbilden (vgl. dazu den Abschnitt zu Coach the Coach).

Im Folgenden sind die zentralen Inhalte und Themengebiete stichwortartig aufgeführt, welche in einer ersten Qualifizierung enthalten sein sollten.

\subsubsection{Grundlagen}

Definitionen und Anwendungsfelder von Beratungsformen allgemein und Coaching im Speziellen, Theoretische Grundlagen des Coachings.

\subsubsection{Coaching-Prozess}

Erstkontakt, Auftragsdynamik, Ziel-, Kontext- und Auftragsklärung, Vertrags- und Prozessgestaltung, Phasen eines Coachings allgemein und einer Sitzung im Speziellen, Abschluss und Transfertechniken, Evaluation.

\subsubsection{Settings}

Externes versus organisationsinternes Coaching, Teamund Gruppencoaching, Coaching im Rahmen von Changeund Organisationsentwicklungsprozessen, Coaching und Komplementärberatung.

\subsubsection{Methoden und Techniken der Intervention}

Methoden entlang des Coaching-Prozesses, Fragetechniken, Selbstreflexions- und Veränderungstechniken, (Problem-)Lösungs- und Entscheidungstechniken, Einsatz von Hypothesen, Feedback, Arbeit mit kreativen Techniken, Medieneinsatz, Shadowing usw.

\subsubsection{Organisationstheoretische Themen im Coaching}

Theorien soziotechnischer Systeme und der Arbeits- und Organisationspsychologie, Coaching im Kontext von verschiedenen Organisationen und Branchen.

\subsubsection{Rollenkonzepte}

Dynamik von Person-Rolle-Organisation, Rollentheorien, Rollenkonzepte des Coach und der Kunden in ihrem Kontext, Rollenkonflikte.

\subsubsection{Psychologische Themen}

Persönlichkeitsentwicklung und Verhaltensänderung, Motivationspsychologie, Arbeit mit Emotionen im Coaching-Prozess, Abgrenzung zur Arbeit im Bereich der „psychischen Störungen“, Indikationsstellungen.

\subsubsection{Berufliche und persönliche Veränderungsprozesse}

Entwicklungspsychologie, Lebens- und Karrierewege, Umgang mit Veränderungen, Führung in Veränderungsprozessen.

\subsubsection{Persönliche und soziale Kompetenzen}

Haltung, Rolle und Menschenbild als Coach, Aufbau und Gestaltung einer wertschätzenden Beziehung, Fähigkeit zur Selbst- und Fremdwahrnehmung und zur Selbstreflexion, Umgang mit persönlichen „blinden Flecken“ und mit anspruchsvollen Situationen im Coaching.

\subsubsection{Führung und Management}

Management- und Führungsmodelle, Reflexion von Führungsverständnis beim Kunden, Führen in anspruchsvollen Situationen (Change, Krise usw.), Führung und Diversity.

\subsubsection{Umgang mit Krisen}

Ganzheitliche Betrachtung von Krisen, Umgang mit Krisensituationen, Stress und Stressmanagement.

\subsubsection{Umgang mit Veränderungen und Konflikten}

Konfliktdiagnose und -bearbeitung, Change Management.

\subsubsection{Professionalisierung im Coaching (Positionierung, Weiterbildung)}

Entwicklung einer eigenen Rahmentheorie und eines Marketing-Konzeptes, Aufbau eines Coaching-Netzwerkes inkl. 
Qualitätssicherung der eigenen Professionalität (Supervision, Intervision).

\subsection{Anforderungen an die Didaktik}

Die Weiterbildung berücksichtigt die Erkenntnisse der Lernpsychologie bezüglich verschiedener Lerntypen und Lernformen der Teilnehmenden (vgl. z. B. Negri 2010). Die Lernziele sollten auf jeden Fall auf allen drei Ebenen (kognitiv, affektiv und psychomotorisch) formuliert und in den Leistungsnachweisen überprüft werden. Im Zentrum steht die Möglichkeit, dass die Teilnehmenden in verschiedenen Formen die Gelegenheit haben, sich als Kunde oder Coach zu erfahren und die eigenen Einstellungen, Werthaltungen, Emotionen und Handlungen in diversen Feedbackschlaufen zu reflektieren. Die kognitiven Lernziele sollten so weit es geht außerhalb der Präsenzkurse verfolgt werden. Dazu sind verschiedene Formen des Selbststudiums geeignet, von der klassischen Lektüre über E-learning (inkl. Visierung von Lehrvideos) bis zum Verfassen von Semester- oder Abschlussarbeiten.

Das Einüben von spezifischen Fähigkeiten geschieht sinnvollerweise in interaktiven Settings. Hier eignen sich vor allem folgende didaktische Formen:

- Referate, Präsentationen, Lehrgespräche mit dazugehörenden Diskussionen

- Einzel-, Paar- und Gruppenarbeiten

- Beratungen in wechselnder Konstellation (Coach-Kunde-Beobachtung), auch kombiniert mit Reflecting Team, Audio- oder Videoaufnahmen

- Rollen- oder Planspiele mit Beobachtung

- Aufstellungen

- Live-Coachings (auch eventuell kombiniert mit Reflecting Teams)

- Fish-Bowl oder Debatten für die Auseinandersetzung mit kontroversen Themen

- Action Learning: Einsatz bei Kunden im Feld

Die interaktiven Kurse finden idealerweise in zwei-bis mehrtägigen Blöcken statt, so dass die Gruppenmitglieder auch in Pausen und am Abend (bei Seminaren mit Übernachtung) informelle Kontakte pflegen können. Außerhalb der Kurssequenzen sind aber auch folgende didaktischen Gefäße notwendig:

- Eigene Beratungsfälle. Während die Stiftung Warentest davon ausgeht, dass die Teilnehmenden mindestens ein Coaching mit einem echten Kunden durchführen sollte, äußert sich der DBVC sehr vage: Ein „Lernprojekt: vereinbartes Lern- und Anwendungsbeispiel“" (2012, S. 85), Rauen und Steinhübel schreiben gar lediglich von „Übungsklienten“ (S. 309), das ist doch sehr unspezifisch und unverbindlich. Der BSO verlangt meh- rere Beratungsprojekte mit mindestens $40 \mathrm{~h}$ direktem Kundenkontakt.

- Lehrsupervision für die Bearbeitung, Besprechung und Reflexion der Beratungsmandate.

- Intervision zur Einübung der Praxisberatung unter Kolleginnen und Kollegen

- Arbeit in Lerngruppen: Die sogenannten Peergruppen dienen dazu, dass die Teilnehmenden sich gegenseitig unterstützen bezüglich Lernstoff (Lektüre, Vor- und Nachbereitung der Kurse, Fallbesprechungen). Im Masterlehrgang am IAP fusionieren in der Hälfte der Weiterbildung diese Gruppen zu Intervisionsgruppen.

- Einzelcoaching bzw. Einzellehrsupervision: Auch dieses Setting sollte vor allem für Teilnehmende obligatorisch sein, welche über keine Erfahrungen in der Kundenrolle verfügen.

\subsection{Abschlussverfahren}

Damit ein Zertifikat oder Diplom ausgestellt werden kann, sind folgende Leistungen wichtig:

- Teilnahme an den Kursen und den anderen didaktischen Gefäßen

- Eigene Beratungsmandate und deren Reflexion in und im Anschluss an die Super- bzw. Intervision

- Schriftliche Abschlussarbeit (idealerweise Verbindung zwischen Beratungsmandat/en und theoretischen Ausführungen)

- Erfolgreiche Teilnahme an einer Schlussqualifikation (Fallkolloquium, Diplomarbeitskolloquium, Klausurarbeit oder andere erwachsenenbildungsgerechte Nachweise).

Die erfolgreiche Teilnahme wird mit einem Zertifikat oder bei längerdauernden Lehrgängen mit einem Diplom ausgewiesen. Diese können dann die Zulassung zu einem entsprechenden Coaching-Verband ermöglichen.

\subsection{Anforderungen an die Dozierenden}

Dozierende in Coaching-Weiterbildungen verfügen als erstes über die Qualifikationen und Kompetenzen von Coachs mit viel Erfahrung in der Praxis. Darüber hinaus sind sie in der Lage, Erkenntnisse aus der Coaching-Forschung und -Literatur in ihr Curricula einzubauen (z. B. über Gastdozierende, Pflichtlektüren usw.). Didaktische Erfahrung (speziell in der Erwachsenenbildung) und Kenntnisse im Umgang mit Gruppen(dynamik) sind zusätzliche Qualifikationen. Die Dozierenden erhalten ihre eigene Leistungsfähigkeit durch ständige Auseinandersetzung mit Theorie und Praxis, entsprechenden Weiterbildungen und Supervision. Sie sind bereit, sich kontinuierlich beurteilen zu lassen, ob es ihnen gelingt, in einem 
angenehmen Lernumfeld ihre Erfahrungs-, Fach- und Methodenkompetenzen zielführend einzusetzen.

\subsection{Anforderungen an die Teilnehmenden}

Neben den Dozierenden bildet die optimale Auswahl der Teilnehmenden einen wesentlichen Bestandteil für das Gelingen einer Weiterbildung zum Coach. Bezüglich Selbstund Sozialkompetenzen sollten die Interessenten bereits recht hohe Ausprägungsgrade mitbringen, auf welche die Lehrgangsleitung beim Auswahlverfahren achten sollte. $\mathrm{Da}$ es sich um eine Weiter- und nicht Grundausbildung handelt, sollten auch Vorkenntnisse zumindest bezüglich Fachinhalten mitgebracht werden. Idealerweise verfügen die Teilnehmenden über ein Hochschulstudium etwa in Psychologie, Sozial- oder Wirtschaftswissenschaften. Natürlich sind Absolventen mit naturwissenschaftlichem oder technischem Hintergrund auch eine wertvolle Bereicherung, ebenso Personen, welche über viel Berufs- und oder Führungserfahrung verfügen. Entscheidend für eine Aufnahme dürften aber in erster Linie die Erfahrung und Bereitschaft zur Auseinandersetzung mit sich und andern Personen sein. Da die Weiterbildung in der Regel berufsbegleitend erfolgt, sollten die Teilnehmenden zusätzlich über ein gutes Selbstmanagement, emotionale Stabilität und Frustrationstoleranz verfügen (vgl. Rauen und Steinhübel 2005).

\subsection{Anforderungen an die Qualitätssicherung}

Die Anbieter evaluieren regelmäßig und systematisch ihre Weiterbildungen und sichern damit die Struktur-, Prozesse und Ergebnisqualität der Lehrgänge. In Anlehnung an Kirkpatrick kann das 4-Stufen-Modell der Evaluation eine hilfreiche Orientierung sein (Kirkpatrick und Kirkpatrick 2006; Künzli 2010):

1. Reaktionen: Erleben der Teilnehmenden: Die Teilnehmenden beurteilen etwa die Inhaltsrelevanz, Didaktik, Formate, Zielklarheit, Dauer, Seminarort, Beziehungsgestaltung usw.

2. Lernen: Erweiterung von Kenntnissen und Kompetenzen: Diese können über Selbst- oder Fremdeinschätzung eingestuft oder gar geprüft werden.

3. Verhalten: Anwendung des Gelernten im relevanten Kontext: Hier geht es um die Umsetzung des Gelernten in der Praxis. Konkret könnten Selbsteinschätzungen, Kundenfeedbacks oder Beobachtungen (im Feld oder per Videoaufnahmen) Anhaltspunkte liefern.

4. Resultate: Betriebswirtschaftlicher Nutzen für den Auftraggeber: Hier geht es um die Einschätzung, ob sich die Investition in die Weiterbildung für den Einzelnen (oder die Organisation, welche sie finanzierte), wirtschaftlich gelohnt hat. „Harte“ Indikationen könnten etwa Bera- tungsmandate sein, „,weiche“ die Kundenzufriedenheit des Weiterbildungsteilnehmenden oder dessen Kunden usw.

Wie intensiv eine Weiterbildung evaluiert werden soll, ist nicht zuletzt abhängig von dessen Umfang. Da die Masterlehrgänge am IAP über zweieinhalb Jahre dauern, werden hier darüber hinaus auch regelmäßige Transferevaluationen durchgeführt, bei denen die Teilnehmenden ca. anderthalb Jahre nach Abschluss nochmals befragt werden, wie sie den Lehrgang nach einer gewissen Zeit einschätzen. Selbst eine Befragung nach mehreren Jahren kann wertvolle Erkenntnisse bringen, vor allem über die Frage, wie viele Teilnehmende den Sprung in die Coaching-Praxis tatsächlich finden (Guggenbühl und Kuhn 2006).

\subsection{Hinweise für die Suche nach einer geeigneten Weiterbildung}

Für die Auswahl einer geeigneten Weiterbildung ist ein erster Schritt die Analyse der eigenen Zielsetzungen und Bedürfnisse (vgl. Rauen und Steinhübel 2005). Dies ermöglicht eine präzisere Suche nach möglichen Anbietern. Dazu können die Websites der wichtigsten Berufsverbände eine erste Hilfe sein, denn diese führen in der Regel die von ihnen anerkannten Weiterbildungen auf. Ähnlich wie bei der Suche nach einem Coach empfiehlt es sich auch bezüglich Weiterbildung die persönlichen Empfehlungen aus dem eigenen Netzwerk zu nutzen (Vogelauer 2013a). Anbieter führen in der Regel auch Informationsveranstaltungen und Vorgespräche durch. Bei einigen besteht die Möglichkeit, eine erste Weiterbildungseinheit unabhängig von der ganzen Weiterbildung zu buchen. Dies ist natürlich bei modularen Curricula einfacher als bei geschlossenen Lehrgängen. Aber zumindest ein Kennenlernen in der Lerngruppe wäre auch da eine Möglichkeit, damit hat der Autor sehr gute Erfahrungen gemacht. Denn die Teilnehmenden schätzen es, wenn sie im Voraus auch wissen, wer sich mit ihnen auf einen längeren und oft auch intensiven Prozess einlässt. Nicht zuletzt bildet eine gute Gruppe auch nach einer Weiterbildung ein wertvolles Netzwerk im Beruf und speziell für die Beratungstätigkeit. Zwei Aspekte seien hier besonders betont: Seriöse Anbieter geben ernsthaften Interessenten auch Referenzen von ehemaligen Teilnehmenden. Diese Quellen sollte man auf jeden Fall nutzen, denn die Sicht der Teilnehmenden ist doch nochmals eine andere als diejenige der Dozierenden und Lehrgangsleitenden. Rauen und Steinhübel empfehlen die Abklärung, ob Supervisionen angeboten und während der Weiterbildung mit „Übungsklienten“ gearbeitet werde. Wie bei den didaktischen Gefäßen bereits betont, wird hier klar die Meinung vertreten, dass sowohl Lehrsupervision wie auch verbindliche Beratungsmandate keine „nice to have“-, sondern absolute Muss-Kriterien sind für eine seriöse Qualifizierung als 
Coach. Niemand möchte zu jemandem ins Coaching gehen mit einem Weiterbildungszertifikat, bei welchem man dessen erster Kunde ist und diese Person im schlechtesten Fall gar nicht mehr in einer Lehrsupervision ist. Damit leite ich zum letzten Abschnitt über. Selbst wenn eine Qualifizierung „abgeschlossen“ ist, so wird ein professioneller Coach sich permanent weiterbilden und seine Arbeit in Super- oder Intervision immer wieder reflektieren.

\section{Und wer coacht den Coach oder wie entwickelt sich Coaching-Qualifizierung weiter?}

„Und wer schützt die Polizei?“ sang der Kabarettist Georg Kreisler einst. Analog dazu kann die Frage gestellt werden, wer in einem Unternehmen für das Human Resource Management schaut oder wer denn die Coachs weiterentwickelt oder berät. Die meisten Berufsverbände verlangen deshalb von ihren Mitgliedern zu Recht, dass sie sich permanent weiterbilden und reflektieren. In Anlehnung an Vogelauer (2013c) können folgende zentralen Formen der Fortbildung für Coachs in Betracht gezogen werden:

- Reflexionscoaching mit einem Coach oder Supervisor („Coach the Coach“ im engeren Sinn)

- Reflexionscoaching in Gruppen (Intervision oder Supervision)

- Vertiefungsworkshops

- Vertiefende Weiterbildungen zum Beispiel bezüglich einem spezifischen Verfahren

- Kongressbesuche

- Austauschforen, Vereine, Verbände

- Studium von Literatur oder Forschungsergebnissen, alleine oder in einer Peergruppe

Generell kann davon ausgegangen werden, dass es in der Qualifikation von Coachs zwei hauptsächliche Entwicklungen geben mag: Auf der einen Seite eher kürzere Weiterbildungen in der Größenordnung der DBCV-Anforderungen, deren Absolventen nur zu einem kleinen Teil als Coach arbeiten werden. Diejenigen darunter, welche sich für Coaching als „Profession“ entscheiden, werden sich permanent weiterqualifizieren. Auf der anderen Seite die länger dauernden Weiterbildungen, welche mindestens den Standard eines BSO oder DGSv erfüllen bis hin zu den akademisierten Studiengängen (vgl. Strikker und Strikker 2013; Grant 2011). Bei letzteren stellt sich die Herausforderung, wie sie den Gap zwischen Experten aus der Praxis und Wissenschaftlern, die sich auf Forschungsergebnisse stützen, fruchtbar überwinden können (Möller et al. 2013). Nicht nur in diesem Feld gibt es noch viel zu tun. Auch bei der Qualifizierung von Fortgeschrittenen ist das Feld weit offen. Als Veranschaulichung dazu dient ein kurzer Beratungs-Ausschnitt aus einem Buch „für Fortgeschrittene“ (Schulte 2013, S. 198): Der
Coachee ist nach Einschätzung des Coachs in seinem negativen Weltbild gefangen, nachdem er frustriert über eine für ihn ärgerliche Reaktion seitens seines Vorstandes berichtet hat. Er zweifelt daran, ob ihm das Coaching was bringe und beschreibt auf die Frage seines Coachs hin das vor ihm stehende Glas als halb leer. Nun folgt ein interessanter Dialog, den ich hier ausnahmsweise wiedergebe:

Coach: „Falsch!“

Kunde: „Wieso?"

Coach: „Das Glas ist halb voll.“

Kunde: „Ach so, das meinen Sie“.

Coach: „Kennen Sie den Unterschied?“

Kunde. „Schon klar, Optimist und Pessimist“.

Coach: „Ja, aber es geht noch weiter. Ein halb leeres Glas ist eine kleine Katastrophe, denn es drückt aus, dass man nicht mehr viel hat. Ein halb volles Glas ist eine Aussage, dass man noch ausreichend zu trinken hat. Das ist ein gewaltiger Unterschied. Das eine ist eine kleine Katastrophe, das andere Quelle der Hoffnung. Ist Ihnen das klar?“"

Darauf folgt ein Dialog etwa Sinngemäß nach dem Motto „das Gute im Schlechten“.

Nun die eingangs bereits gestellte Frage zum Schluss: Ist das Glas halb voll oder halb leer, wenn wir uns über die Qualifizierung von (fortgeschrittenen) Coachs unterhalten? Wer soll bei solchen Fragen die Deutungshoheit haben? Gerne rege ich dazu eine andere Sichtweise an: Ein Glas ist in der Regel weder halb voll noch halb leer. Es kann nämlich durchaus auch voll sein: Wenn es teilweise mit Wasser gefüllt ist, dann besteht der Rest der Füllung mit hoher Wahrscheinlichkeit aus Luft, und ob diese kalt, warm oder heiss ist, hängt auch wieder von der Perspektive des Betrachters ab. Ist Ihnen das klar? Meine Ironie bezieht sich hier nicht nur auf das Zitat aus dem genannten Buch, sondern auch auf dessen Untertitel (,für Fortgeschrittene“!).

Und ob Luft oder Wasser fürs Überleben wichtiger ist, sei ebenfalls dahingestellt. Da füge ich ergänzend die von mir bereits vor einigen Jahren (Lippmann 2007) gestellte Frage abschließend an: „Alles Coaching (auch für Fortgeschrittene) ....oder was?" Die Anlehnung an einen Werbespruch scheint ja anregend zu sein (Loebbert 2013). Auf weitere Auseinandersetzungen können wir alle gespannt sein, auch wenn manchmal etwas „heisse Luft“ dabei sein mag.

Open Access Dieser Artikel wird unter der Creative Commons Namensnennung 4.0 International Lizenz (http://creativecommons. org/licenses/by/4.0/deed.de) veröffentlicht, welche die uneingeschränkte Nutzung, Verbreitung und Wiedergabe für beliebige Zwecke erlaubt, sofern Sie den/die ursprünglichen Autor(en) und die Quelle ordnungsgemäß nennen, einen Link zur Creative Commons Lizenz beifügen und angeben, ob Änderungen vorgenommen wurden. 


\section{Literatur}

Berufsverband für Coaching, Supervision und Organisationsberatung (BSO). (2013). Formale Mindestanforderungen und Eingangsvoraussetzungen. http://www.bso.ch/verband/vertragl-ausbildungspartnerschaft.html. Zugegriffen: 15. Feb. 2014

Böning, U., \& Fritsche, B. (2008). Coaching fürs Business (2. Aufl.). Bonn: managerSeminare.

Brandenberger, T., \& Gassmann, N. (2006). Kompetenter Coach? Erwartete Kompetenzen aus der Sicht von Organisationen (2. Studienaarbeit). Zürich: Hochschule für Angewandte Psychologie.

Deutsche Gesellschaft für Supervision (DGSv). (2013) Standards für die Qualifizierung zur/zum Supervisor/in. http://www.dgsv.de/ supervisorin-werden/. Zugegriffen: 15. Feb. 2014

Deutscher Bundesverband Coaching e.v. (DBVC). (Hrsg.). (2012). Leitlinien und Empfehlungen für die Entwicklung von Coaching als Profession (4. erw. Aufl). Osnabrück: Eigenverlag.

Erpenbeck, J., \& von Rosenstiel, L. (2007). Einführung. In J. Erpenbeck \& L. von Rosenstiel (Hrsg.), Handbuch Kompetenzmessung - Erkennen, verstehen und bewerten von Kompetenzen in der betrieblichen, pädagogischen und psychologischen Praxis (2. erw. Aufl.) (S. IX-XL). Stuttgart: Schäffer-Poeschel.

Gassmann, D., \& Grawe, K. (2006). General change mechanisms: The relation between problem activation and resource activation in successful and unsuccsessful therapeutic interactions. Clinical Psychology \& Psychotherapy, 13(1), 1-11.

Geissler, H. (2011). Coaching meets Training - zur Lösung des Transferproblems durch „virtuelles Transfercoaching (VTC)“. In R. Wegener, A. Fritze \& M. Loebbert (Hrsg.), Coaching entwickeln. Forschung und Praxis im Dialog (S. 123-134). Wiesbaden: VS Verlag für Sozialwissenschaften.

Grant, A. M. (2011). Developing an agenda for teaching coaching psychology. International Coaching Psychology Review, 6(1), 84-99.

Greif, S. (2008). Coaching und ergebnisorientierte Selbstreflexion. Göttingen: Hogrefe.

Greif, S. (2009). Grundlagentheorien und praktische Beobachtungen zum Coachingprozess. In B. Birgmeier (Hrsg.), Coachingwissen. Denn sie wissen nicht, was sie tun? (S. 129-144). Wiesbaden: VS Verlag für Sozialwissenschaften.

Greif, S., Schmidt, F., \& Thamm, A. (2012). Warum und wodurch Coaching wirkt. Ein Überblick zum Stand der Theorieentwicklung und Forschung über Wirkfaktoren. Organisationsberatung, Supervision, Coaching, 4, 375-390.

Guggenbühl, B., \& Kuhn, C. (2006). IAP-Diplomstudiengang Supervision und Coaching in Organisationen. Berufserfahrungen von ehemaligen Absolventinnen und Absolventen (unveröffentl. 2. Studienarbeit im Rahmen des Nachdiplomstudiengangs in Berufs- und Laufbahnberatung). Zürich: Institut für Angewandte Psychologie.

Kaufel, S., Scherer, S., Scherm, M., \& Sauer, M. (2006). Führungsbegleitung in der Bundeswehr. Coaching für militärische Führungskräfte. In W. Backhausen \& J.-P. Thommen (Hrsg.), Coaching. Durch systemisches Denken zur innovativen Personalentwicklung (S. 419-438). Wiesbaden: Gabler.

Kauffeld, S. (2006). Kompetenzen messen, bewerten, entwickeln. Ein prozessanalytischer Ansatz für Gruppen. Stuttgart: Schäffer-Poeschel.

Kirkpatrick, D. L., \& Kirkpatrick, J. D. (2006). Evaluating training programs. The four levels. San Francisco: Berrett-Kohler.

Kuchen, C., \& Pedrun, P. (2006). Welche Kompetenzen braucht ein Coach? Das ideale Kompetenzprofil aus Sicht von Coachs (unveröffentl. 2. Studienarbeit). Zürich: Hochschule für Angewandte Psychologie.

Kuhl, J. (2001). Motivation und Persönlichkeit. Interaktionen psychischer Systeme. Göttingen: Hogrefe.
Künzli, H. (2010). Evaluation von Lernprozessen - Wirksamkeit, Wirtschaftlichkeit und Nachhaltigkeit. In C. Negri. (Hrsg.), Angewandte Psychologie für die Personalentwicklung. Konzepte und Methoden für Bildungsmanagement, betriebliche Aus- und Weiterbildung (S. 264-279). Heidelberg: Springer.

Lippmann, E. (2007). Alles Coaching, ... oder was? Forum Supervision, 29, 26-39.

Loebbert, M. (2013). Alles Coaching oder was? Coaching in unterschiedlichen Praxisfeldern. Coaching-Magazin, 3, 48-51.

Meier, R., \& Janssen, A. (2011). CoachAusbildung - ein strategisches Curriculum (2. überarb. Aufl.). Sternenfels: Verlag Wissenschaft \& Praxis.

Merz, C., \& Frey, A. (2011). Empirisch fundiertes Kompetenzmodell für den Bereich des Führungskräftecoachings. Aachen: Shaker Verlag.

Möller, H., Kotte, S., \& Oellerich, K. (2013). Coaching-Praxis und Wissenschaft - ein unüberwindlicher Gap? Coaching-Magazin, 1, 35-39.

Negri, Ch (Hrsg.). (2010). Angewandte Psychologie für die Personalentwicklung. Konzepte und Methoden für Bildungsmanagement, betriebliche Aus- und Weiterbildung. Heidelberg: Springer.

Rauen, Ch, \& Steinhübel, A. (2005). Coaching-Weiterbildungen. In Ch Rauen (Hrsg.), Handbuch Coaching (S. 289-310). Göttingen: Hogrefe.

Schulte, T. (2013). Der Weg zum professionellen Coach. Coaching für Fortgeschrittene. Weinheim: Beltz.

Stiftung Warentest (2013). Coachen im beruflichen Kontext. Was eine gute Einstiegsqualifizierung bieten sollte. http://www.test.de/Coaching-Lehrgaenge-Coachen-will-gelernt-sein-4604619-0/. Zugegriffen: 27. Sept. 2013

Strikker, H., \& Strikker, F. (2013). Coaching studieren? Akademisierung im Business-Coaching. Coaching-Magazin, 2, 36-41.

Vogelauer, W. (2013a). Was macht einen professionellen Coach aus? In W. Vogelauer. (Hrsg.), Coaching-Praxis. Das Trigon-Modell: Konzept und Methoden (S. 149-158). Weinheim: Beltz.

Vogelauer, W. (2013b). Ergebnisse der sechsten Coaching-Befragung 2012. In W. Vogelauer. (Hrsg.), Coaching-Praxis. Das Trigon-Modell: Konzept und Methoden (S. 159-182). Weinheim: Beltz.

Vogelauer, W. (2013c). Coach the Coach: Weiter- und Fortbildung von Profis. In E. Lippmann (Hrsg.), Coaching. Angewandte Psychologie für die Beratungspraxis (S. 304-315). Heidelberg: Springer.

Winkler, B., Lotzkat, G., \& Welpe, I. M. (2013). Wie funktioniert Führungskräfte-Coaching? Orientierungshilfe für ein unübersichtliches Beratungsfeld. OrganisationsEntwicklung, 3, 23-33.

\section{Further reading}

Kühl, S. (2006). Die Supervision auf dem Weg zur Profession? Organisationsberatung, Supervision, Coaching, 13, 5-18.

Lippmann, E. (2009). Meisterschaft erlangen in Coaching - Der Masterlehrgang am Institut für Angewandte Psychologie in Zürich. In J. Dahinden, Th Freitag, \& F. Schellenberg (Hrsg.), Mythos Coaching. Was bringt's? Wie funktioniert es? (S. 166-177). Zürich: Orell Füssli.

Lippmann, E. (Hrsg.). (2013). Coaching. Angewandte Psychologie für die Beratungspraxis (3. Aufl.). Heidelberg: Springer.

Österreichischer Dachverband für Coaching (2007/2008). Lehrgangsinhalte. http://www.coachingdachverband.at/index_html?id=225. Zugegriffen: 15. Feb. 2014.

Schmidt, G. (2013a). Liebesaffären zwischen Problem und Lösung: Hypnosystemisches Arbeiten in schwierigen Kontexten (5. Aufl.). Heidelberg: Carl Auer.

Schmidt, G. (2013b). Vorwort. In S. Radatz (Hrsg.), Beratung ohne Ratschlag (8. Aufl., S. 13-23). Wien: Verlag systemisches Management. 